\title{
Sobre la reinterpretación de algunos procesos derivativos en la Base de datos morfológica del español (BDME)
}

\section{On the reinterpretation of some derivational processes in the Morphological Database of Spanish (BDME)}

\author{
Jesús Pena \\ Universidade de Santiago de Compostela
}

Resumen. La Base de datos morfológica del español (BDME) está construida desde una perspectiva diacrónica. Sus características y estructura están descritas en Pena (2018). El objetivo de esta contribución es explicar las principales novedades adoptadas en la tipología de procesos, consistentes en (i) el añadido de dos tipos, regresión y moción genérica, (ii) modificaciones en la aplicación del proceso de conversión y (iii) la interpretación del cruce léxico.

Palabras clave: base de datos morfológica, familia de palabras, procesos morfológicos.

Abstract. The Base de datos morfológica del español 'Morphological database of Spanish' $(B D M E)$ has been constructed along diachronic lines. Its characteristics and structure have been described in Pena (2018). The aim of this contribution is to show the main innovations of the database with respect to the typology of processes, which consist of (i) the addition of two types, i.e. backformation and gender motion, (ii) modifications in the application of the process of conversion and (iii) the interpretation of blending.

Keywords: morphological database, word family, morphological processes.

Data de recepción: 29-05-2020 - Data de aceptación: 08.07.2020. 
LA BASE DE DATOS MORFOLÓGICA DEL ESPAÑOL (BDME) es una base de datos construida desde una perspectiva histórico-diacrónica, pero solo puede ser parcialmente histórica mientras no se disponga de un diccionario histórico completo y actualizado donde se describa la evolución del léxico español a lo largo del tiempo, esto es, la evolución fonética, morfológica y semántica de cada palabra desde su origen. Lo que ofrece la $B D M E$ son familias de palabras relacionadas morfológicamente y emparentadas genéticamente. Se trata, por tanto, de una base de datos morfoetimológica, en la medida en que se fija prioritariamente en la construcción morfológica de la palabra teniendo en cuenta su origen inmediato y último (véase Cottez 1980) ${ }^{1}$.

La $B D M E$ está diseñada para establecer, organizar y mostrar las relaciones etimológicas y derivativas entre las palabras del español. En Pena (2018) se presenta la configuración general de la base de datos y se describe en detalle cada una de las propiedades analizadas al introducir una palabra. En dicho trabajo se examinan los siguientes aspectos: a) el concepto de familia léxica y sus fundamentos morfológicos; b) las unidades necesarias para establecer la estructura de la palabra; c) la oposición entre palabra y tema; d) la interpretación de los diferentes procesos morfológicos; e) las dos formas de representar las relaciones morfoetimológicas en la aplicación web que se nutre de los datos almacenados en la BDME: diagramas arbóreos e imagen lineal (https://bdme.iatext.es) ${ }^{2}$.

El objetivo de esta breve contribución es explicar los aspectos novedosos que atañen a la tipología de procesos presentada en Pena (2018: §3.2.2.3). Las decisiones adoptadas en cuanto al catálogo de procesos y a su aplicación al análisis de las palabras complejas han requerido la lectura crítica de varios trabajos y una toma de postura que, en el caso de los cambios donde interviene la vocal del tema, implica reinterpretar relaciones derivativas previamente concebidas como sustitución o adición (Pena 1991, 1999, 2018), que pasan a etiquetarse como conversión con cambio de clase flexiva o de tema. Para revisar los procesos que han sido objeto de alguna modificación, conceptual o terminológica, se seguirá el siguiente orden: regresión, sustitución, conversión, moción genérica, contaminación y cruce léxico.

REGRESIÓN. El proceso conocido como regresión (ingl. backformation, al. Rückbildung, fr. dérivation régressive, it. retroformazione) nace en la nomenclatura lingüística como opuesto a la derivación progresiva, que es la afijación, y surge como

Como me señala un evaluador anónimo, es importante insistir en el carácter diacrónico de la base de datos, ya que el criterio que justifica la dirección derivativa de los procesos morfológicos es histórico. Solo así se comprende que en la BDME tengan diferente tratamiento comprar $>$ comprador y legislador > legislar.

2 Este localizador de la aplicación web es de 2020; las direcciones anteriores, proporcionadas en Pena (2018), redirigen a esta última. En el primer trimestre de 2020 se ha obtenido para el recurso en línea el siguiente ISSN: 2660-7190. 
un proceso morfológico que utilizan ocasionalmente los hablantes para dar cuenta de palabras complejas cuya particularidad consiste en carecer de base de derivación. Se define como proceso que consiste en «invertir» la dirección de una regla productiva de formación de palabras (Rainer 2004b: 495); por tanto, en la regresión una palabra formalmente igual a otras afijadas experimenta una operación de desafijación: el lat. collā-re, formado sobre collā-tio, opera mediante regresión frente a la regla productiva de sufijación creā-re $\rightarrow$ creā-tio; otro tanto sucede con el esp. legisla-r formado por regresión de legisla-dor, frente a la regla de sufijación compra-r $\rightarrow$ compra-dor, o la regresión del ingl. edit-or $\rightarrow$ edit frente a la sufijación exhibit $\rightarrow$ exhibit-or.

Como se puede observar, el proceso de regresión equivale al proceso de afijación invertido; en estas formaciones se opera con el mismo afijo, sufijo en este caso, tomando como base de derivación el segmento más corto en la afijación y el más largo en la regresión ${ }^{3}$. En lenguas de morfología predominantemente aditiva como el español, la regresión funciona como proceso supletorio de la afijación y opera como proceso morfológico para rellenar lagunas en las series de derivación; pero, una vez creada la palabra mediante este proceso y afianzada en el uso $(\mathrm{N} \rightarrow \mathrm{V}$ : legislador $\rightarrow$ legislar), se reinterpreta o reanaliza sincrónicamente como palabra base de derivación $(\mathrm{V} \rightarrow \mathrm{N}$ : legislar $\rightarrow$ legislador, al igual que ocurre en comprar comprador, vender vendedor, etc. (Pena 1991: 112-114).

En algunos casos las formaciones regresivas se reconocen sincrónicamente por el hecho de que no obedecen a patrones regulares de la lengua; por ejemplo, ante el par ilimitar $\leftarrow$ ilimitado se debe proponer la dirección indicada porque el prefijo negativo in- es un prefijo deadjetival, no deverbal; lo mismo sucede con el par boquiabrir(se) $\leftarrow$ boquiabierto: se debe proponer la dirección indicada porque el morfema de unión - $i$ - es típico de adjetivos compuestos formados de nombre + adjetivo, no de compuestos verbales, cf. cuellicorto, cariancho, etc. (Rainer 1993: 82). En otras ocasiones, sin embargo, las regresiones son acordes con el sistema morfológico vigente en un estado sincrónico, pero disconformes con el origen de la palabra (latín, por ejemplo). Y es este desvío etimológico el que permite detectar una regresión. Rainer (2004b: 496) reconstruye el hipótetico ${ }^{\circ}$ ablare a partir de ablazione y sostiene que debe ser una regresión (retroformazione) «perché sappiamo che il verbo base di lat. ablatio, l'etimo di ablazione, era auferre e non *ablare, mai esistito in latino». Otro tanto se puede decir de legislador en español, cuyo origen es el latín legislator; esta lengua no dispone del correspondiente verbo que, de haber existido, hubiese sido olegisferre, no *legislare, por ser el latín lātor, lo mismo que lātio, sustantivos deverbales correspondientes a ferre, a través del participio lātus, $-a,-u m$.

Se pregunta un evaluador si en legislar no hay sufijación alguna (ni sincrónica ni diacrónicamente). Creo que no, por la razón apuntada al final del párrafo siguiente. 
Sustitución. El proceso de sustitución (ing. replacement, al. Substitution, it. sostituzione) consiste en crear una palabra sobre una base de derivación de igual grado de complejidad (Rainer 1993: 171-172, 569, 656, 659). Este proceso opera fundamentalmente en la sustitución afijal y temática. En la sustitución afijal interviene sustituyendo a un prefijo (lat. conuergere $\rightarrow$ lat. mod. dìuergere, fr. amarrer $\rightarrow$ démarrer, esp. ant. recudir $\rightarrow$ acudir, bifurcación $\rightarrow$ plurifurcación) y, sobre todo, a un sufijo (fr. anglicisme $\rightarrow$ angliciste, esp. centrismo $\rightarrow$ centrista, adición $\rightarrow$ aditivo, premonición $\rightarrow$ premonitorio). El campo preferido de la sustitución de temas es el de la composición, cuando el segundo tema, que funciona como núcleo, es un tema de palabra inexistente de origen latino y griego fundamentalmente y que atañe, por poner solo un ejemplo, a la relación entre el nombre de la 'disciplina o especialidad' y el nombre del 'especialista', cf. biología $\rightarrow$ biólogo, -a, pediatría $\rightarrow$ pediatra, agricultura $\rightarrow$ agricultor, geografía $\rightarrow$ geógrafo, $a$, etc. En Rainer (1993: 80-81 y 694-697) aún se sostiene que en estos compuestos opera el proceso de sustracción (Subtraktion) de -ia, pero en Rainer (2004a: 10-11) se argumenta a favor de interpretar estos compuestos temáticos como sustitución de temas. En la $B D M E$ optaremos también por este análisis, puesto que nos parecen convincentes los argumentos en los que apoya Rainer (2004a) esta reinterpretación.

CONVERSIÓN (ing. conversion, al. Konversion, fr. conversion, it. conversione). La conversión es un proceso morfológico que relaciona palabras formalmente iguales, que difieren en cuanto a la clase de palabras a la que se adscriben. Para su definición, se puede partir de la que proporciona la gramática de referencia del inglés (Quirk et al. 1980: 1009): «Conversion is the derivational process whereby an item is adapted or converted to a new word-class without the addition of an affix». La conversión es frecuente en las lenguas germánicas, para las que precisamente se ha delimitado este proceso, cf. (to) doubt (V) «dudar» $\rightarrow$ (the) doubt $(\mathrm{S})$ «duda», group $(\mathrm{S})$ «grupo» $\rightarrow$ $($ to $)$ group $(\mathrm{V})$ «agrupar». En los estudios tradicionales sobre la formación de palabras en las lenguas románicas no se utiliza esta denominación, pero sí en la morfología derivativa actual, cf. esp. comprar $\rightarrow$ compra ${ }^{4}$, lija $\rightarrow$ lijar; it. purgare $\rightarrow$ purga, pittura $\rightarrow$ pitturare; port. pagar $\rightarrow$ paga, arma $\rightarrow$ armar, etc.

Se trata de un proceso de difícil delimitación, sobre el que hay mucha controversia, especialmente en cuanto a su naturaleza y a su ámbito de aplicación (Pena 1991: 103-112; Rainer 1993: 74-80; Thornton 2004b: 499-533). En la BDME seguiré las

Se pregunta un evaluador si esta afirmación vale igual para compra en su sentido de 'acción' que para compra en su sentido de 'efecto o resultado'. En mi opinión, el segundo sentido es una extensión semántica, pues carece de lectura eventiva. Por otra parte, el sentido resultativo no es aplicable a un derivado de un predicado de adquisición, como comprar, por mucho que en el $D L E$ se abuse de la fórmula «acción y efecto de V», que evitan otros diccionarios de referencia, como el DUE o el $D E A$. Me he referido a esta cuestión en varias publicaciones, la más reciente Pena (2019). A ella remito para ver mi interpretación de las extensiones semánticas. 
pautas indicadas en Rainer $(1993,1999,2008,2012)$ y en Rainer \& Wolborska-Laurer (2012), por ser este autor el que más ha profundizado en las características de este proceso en español. Tendré muy en cuenta, además, el análisis de Thornton (2004b), la estudiosa que, de manera coherente y sistemática, mejor ilustra los diversos tipos de conversión existentes en una lengua románica como el italiano.

Dos son las características que suelen destacarse de este proceso: entre doubt, verbo y doubt sustantivo hay homonimia porque no hay ningún cambio desde el punto de vista formal y solo el contexto sintáctico permitirá desambiguar si es verbo o sustantivo. Ante la ausencia de criterios formales para determinar el sentido de la derivación, Marchand $\left(1964,1969^{2}\right)$ propone recurrir a un criterio semántico: el significado de la palabra derivada presupone el significado de la palabra base, pero no viceversa. Así, se puede describir el significado de to doubt sin hacer uso de the doubt, y lo mismo sucede con el significado del español comprar con relación a compra, del significado del italiano purgare con relación a purga, etc.

De acuerdo con los tipos de conversión distinguidos por Thornton (2004b), se puede decir que el ámbito de aplicación del proceso de conversión es igual al de la sufijación heterogénea (afecta a las clases léxicas sustantivo, adjetivo, verbo y adverbio) y con la misma función: cambiar la clase (o subclase) de palabras. La novedad que subraya de manera sistemática Thornton es que la clase flexiva de la palabra base es diferente de la clase flexiva de la palabra derivada mediante conversión ${ }^{5}$. Esta autora tiene que reconocer, sin embargo, que, si bien es completamente distinta la flexión verbal de la nominal (cf. it. purgare $\rightarrow$ purga, pittura $\rightarrow$ pitturare, esp. comprar $\rightarrow$ compra, lija $\rightarrow$ lijar), las desinencias flexivas de nombres y adjetivos son en gran medida homónimas (cf. it. detergente/i $\rightarrow$ un detergente/due detergenti, Argentina $\rightarrow$ argentin-o-/i, -a/-e, esp. tranquilizante-/s $\rightarrow$ un/dos tranquilizante/-s, Abisinia $\rightarrow$ abisini-o/-s, $-a /-s$ ), aunque cabe considerar —y la autora así lo hace - el distinto carácter de la flexión en uno y otro caso: inherente en el nombre, contextual, es decir, inducida por una regla sintáctica, en el adjetivo. Hay, además, características sintácticas y semánticas que, de una manera indirecta, permiten detectar el carácter adjetival o nominal de la palabra: la posibilidad de aparecer o no en posición predicativa con el artículo indeterminado, la modificación adverbial o adjetival, la posibilidad de gradación o no, la adverbialización con el sufijo -mente, etc.

Como indica Thornton (2004b: 503), la novedad es relativa pues consta en la obra de Henry Sweet, acuñador del término en inglés. La autora también señala que la noción de 'clase flexiva' está presente en Aronoff (1994). Una de las fuentes más útiles para el fenómeno de la conversión sigue siendo Marchand (1969: 359-390). Este estudioso también indica que Sweet fue el primero en usar el término conversión: «in English, as in many other languages, we can often convert a word, that is, make it into another part of speech without any modification or addition, except, of course, the necessary change of inflection» (Sweet 1900: 38; apud Don, Trommelen \& Zonneveld 2000: 944). 
Presenta una homonimia más fuerte la conversión intracategorial de nombres en nombres, cf. en it. informatica 'disciplina' $\rightarrow$ informatico 'especialista', pues existe homonimia parcial entre la flexión del nombre de la disciplina y la flexión de la especialista en dicha disciplina, al compartir la clase flexiva -a/e. Lo mismo sucede en español, como puede verse al comparar «estudia informática» con «la informática danesa». En pares como it. la mela/il melo o esp. la manzana/el manzano, la flexión permite diferenciar el nombre del «fruto» del nombre del «árbol», pero no ocurre así con el it. il cedro o el esp. el anacardo donde hay homonimia total en cuanto a la flexión ${ }^{6}$.

Según Rainer (2012: 369), el proceso de conversión, además de ser de difícil interpretación, también es de difícil detección en diccionarios y corpus, frente a la afijación, por carecer de marcas explícitas. En los trabajos recién citados de este autor, se mencionan diversos tipos de conversión. Entre los casos de sustantivación de adjetivos mediante conversión propone incluir Rainer los adjetivos étnicos convertidos en nombres de lenguas: (Italia) $\rightarrow$ italiano, $-a \rightarrow$ el italiano, los adjetivos de persona convertidos en sustantivos mediante tipificación o reconocimiento de cualidades definitorias: un hijo superdotado $\rightarrow$ un superdotado, y la conversión de nombres de «disciplina» en nombres de «especialistas en la disciplina»: fisica, gramática $\rightarrow$ un fisico, un gramático (Rainer 1993: 680-681)7. Entre los casos de adjetivación de sustantivos mediante conversión, considera los adjetivos étnicos: Abisinia $\rightarrow$ abisinio, $-a$; los adjetivos relacionales correspondientes a nombres de lenguas: el bable $\rightarrow$ el vocalismo bable 'del bable', a nombres comunes: movimiento ciudadano 'de los ciudadanos', o a nombres agentivos en -ero,-dor e -ista: sindicato enfermero ('de los enfermeros'/* 'de los enfermos'), actividad reportera 'de reportero'; combatía sus ansias fumadoras 'de fumador'; labor coleccionista 'de coleccionista', etc.

Para terminar con la descripción de este proceso, quiero justificar el cambio de posición respecto a la versión de la $B D M E$ descrita en Pena (2018). Decía entonces que seguía las pautas indicadas por Rainer en varios de sus trabajos, y añadía:

la única diferencia reside en que restringiré su ámbito de aplicación a las clases sustantivo y adjetivo, pues en la distinción entre verbo y nombre (sustantivo o adjetivo) sigo,

$6 \quad$ Para estos casos de homonimia total, Thornton (2004b: 507) deja abiertas dos posibilidades de análisis en función del punto teórico adoptado: como extensiones metonímicas o metafóricas, o como conversiones de carácter periférico.

7 Deben descartarse del proceso morfológico de conversión las sustantivaciones elípticas resultantes de eludir el núcleo originario de un sintagma nominal: línea curva $\rightarrow$ la curva, mano derecha $\rightarrow$ la derecha, letra misiva $\rightarrow$ la misiva. Se trata de un proceso diacrónico de lexicalización. Muchos de los sustantivos del español ya lo son en latín mediante sustantivación elíptica: fábrica $<$ lat. fabrica por elisión del núcleo de ars fabrica «arte del obrero», estio < lat. aestiuum por elisión del núcleo de aestîuum tempus «estación veraniega». 
como se acaba de indicar arriba, distinto criterio e interpretación. Rainer interpreta la formación de verbos con - $a$ - (activo, $-a \rightarrow$ activar, ambiente $\rightarrow$ ambientar, escalón $\rightarrow$ escalonar) como conversión y en la $B D M E$ la interpreto como sustitución o adición de la vocal del tema; por otro lado, en los nombres deverbales con vocal átona (comprar $\rightarrow$ compra, cambiar $\rightarrow$ cambio, cesar $\rightarrow$ cese) Rainer habla de los sufijos átonos $-a$, $-e,-o$, mientras que en la $B D M E$ se aplica el proceso de sustitución de la vocal del tema verbal por la vocal del tema nominal (Pena 2018: 39-40).

Efectivamente, en Pena (2018: 38-39) sostenía que en español y en otras lenguas románicas y, en parte, ya en latín, hay un proceso morfológico específico cuya singularidad radica en que solo interviene la VOCAL DEL TEMA (nominal o verbal) para crear el nuevo nombre o verbo. Compárense las series siguientes:

a. $\quad \mathrm{V} \rightarrow \mathrm{S}$ : comprar $\rightarrow$ compra, cambiar $\rightarrow$ cambio, cesar $\rightarrow$ cese.

$\mathrm{S} \rightarrow \mathrm{V}$ : lija $\rightarrow$ lijar, abanico $\rightarrow$ abanicar, alambre $\rightarrow$ alambrar.

$\mathrm{A} \rightarrow \mathrm{V}:$ aparente $\rightarrow$ aparentar.

b. $\quad \mathrm{S} \rightarrow \mathrm{V}$ : almidón $\rightarrow$ almidonar, cincel $\rightarrow$ cincelar.

$\mathrm{A} \rightarrow \mathrm{V}:$ azul $\rightarrow$ azular, igual $\rightarrow$ igualar.

Aunque en (a) opera el proceso de sustitución y en (b) el de adición, el constituyente mórfico del significante a través del cual se crea la nueva palabra es la VOCAL DEL TEMA (VT): si la nueva palabra es un nombre, intervienen las tres vocales, si es un verbo solo interviene la vocal del tema - $a$-, la única productiva. En los casos de comprar $\rightarrow$ compra y lija $\rightarrow$ lijar, las dos vocales - $a$-, la nominal y la verbal, son homónimas; para salir de dudas, basta comparar dos formas flexivas como compras, 'plural' del sustantivo y compras, 'segunda persona de singular del presente de indicativo' del verbo. Las dos vocales $-a$ - son homónimas, pues determinan distintas clases flexivas o se combinan con exponentes de distintas categorías flexivas: los propios de la clase nombre y los propios de la clase verbo. Lo mismo cabe afirmar de las dos vocales latinas - $a$ - presentes en el nombre rosa, ae y en el verbo amāre.

El latín también utiliza la vocal del tema en la formación de verbos. Bajo un criterio formal, se acostumbra a distinguir en latín dos tipos de derivación verbal: la inmediata, con la simple adición de la vocal del tema (dōnum $\rightarrow$ dōn-ā-re), y la mediata, en la que la vocal del tema va precedida de un sufijo formando un grupo sufijal (amārus $\rightarrow$ amāar-icā-re). De las tres vocales, $-\bar{a}$ - es la más productiva a lo largo de toda la latinidad, $-\bar{e}$ - solo es productiva en el latín arcaico, e - -i- en el latín tardío especialmente (Pena 1980: 11-20).

$\mathrm{S} \rightarrow \mathrm{V}:$ bellum $\rightarrow$ bellāre, flōs $\rightarrow$ flōrēre, custōs $\rightarrow$ custōdīre

$\mathrm{A} \rightarrow \mathrm{V}$ : aequus $\rightarrow$ aequāre, albus $\rightarrow$ albēre, inānis $\rightarrow$ inānīre 
El latín no utiliza, en cambio, la VT para la formación de nombres deverbales: los clásicos fuga, pugna y los tardíos destina, falla, proba, roga, computus y rogus no bastan como precedentes directos de la gran cantidad de formaciones romances de este tipo. Un resumen bastante detallado sobre los posibles orígenes de estos nombres deverbales en español puede verse en Pena (1980: 191-203).

Tras un intercambio de opiniones con Franz Rainer, he optado por reinterpretar los tipos anteriores, tanto en español como en latín, como «conversión con cambio de clase flexiva o de tema»: 1) cf. esp. afrontar $\rightarrow$ afruenta $\rightarrow$ afrenta, «conversión con cambio de clase flexiva o de tema (-a)», 2) abanico $\rightarrow$ abanicar «conversión con cambio de clase flexiva o de tema -a-(r)», 3) lat. damnum $\rightarrow$ damnāre "conversión con cambio de clase flexiva o de tema $-\bar{a}-(\mathrm{re})\rangle^{8}$. Y es que, en lo que atañe al cambio de flexión, el comportamiento de las lenguas germánicas — especialmente el inglés, lengua donde más se ha estudiado la conversión-, no parece que tenga mucho que ver con lo que sucede en el latín y las lenguas románicas. Así lo destacan Don, Trommelen \& Zonneveld (2000) a propósito del latín, español y francés, por ejemplo, y ello sin contar con las lenguas no indoeuropeas:

Outside this language group (i.e. Germanic languages), the phenomenon is seriously underresearched, and there may at least be two reason for this: first, the characteristics of the language in question may be such that conversion simply does not or cannot exist; but also, too little is simply known about (the morphologies of) so many languages that it is difficult to draw conclusions on the potential existence of conversion in them [...]. As an example of the former situation, consider a Romance language such as Spanish, where verbs and many nouns take so-called theme-vowels added to their stems, which makes it difficult to talk about conversión in the same way one does with respect to English (Don, Trommelen \& Zonneveld 2000: 950, subrayado mío) ${ }^{9}$.

MOCIÓN GENÉRICA El proceso denominado moción genérica, tomado del alemán Motion o Movierung ${ }^{10}$, se utiliza ahora en la BDME para etiquetar la derivación de sustantivos que designan seres humanos y animados (i.e. nombres comunes de personas y de animales) de determinado sexo a partir del nombre que designa el ser de la misma especie, pero del sexo opuesto. Normalmente la dirección en la moción genérica hace referencia a la formación de sustantivos de referente sexual femenino a partir de los masculinos correspondientes (abad > abadesa, poeta $>$ poetisa, doctor $>$ doctora, camello

\footnotetext{
$8 \quad$ Agradezco la nueva redacción propuesta por uno de los evaluadores.

$9 \quad$ Véase también Valera (2014: 155-168).

10 Véase Rainer (1993: 209-211), Thornton (2004a: 218). Hasta donde conozco, dentro de la tradición gramatical de la RAE, solo figura en el Esbozo de 1973. No consta en la edición de 1931.
} 
$>$ camella, pato $>$ pata, etc.). Aunque esto sucede en la gran mayoría de los casos, no siempre es así, cf. azafata $>$ azafato, prostituta $>$ prostituto, viuda $>$ viudo o rana $>$ rano.

Restringimos la moción genérica a la que ocurre dentro del sustantivo: la adición o sustitución de $-a$, único sufijo productivo, y los no productivos de origen griego o latino: -isa -esa, -ina y -triz. Se descartan, por tanto, los procedimientos sintácticos como los del género común de la gramática tradicional (el/la consorte, mártir). En mi opinión, en Rainer (1993: 210-211) se ofrecen argumentos sólidos para defender el estatuto de morfema derivativo (no flexivo) de la - $a$ y demás sufijos en nombres que se refieren a personas o animales ${ }^{11}$. Para ello conviene diferenciar, como hacen el propio Rainer (1993: 210-211) o la NGLE (2009: 24) para el español, y subraya Thornton (2004a: 219) para el italiano, entre género como propiedad gramatical inherente del sustantivo y sexo (o género natural) que sirve para diferenciar en los seres animados el sexo del referente (gato/gata, alcalde/alcaldesa).

La moción genérica afecta también, claro está, a los nombres de persona que desempeñan un oficio, y que se agrupan bajo la etiqueta «agente clasificador de la acción» cuando se trata de sustantivos deverbales $(\mathrm{V}>\mathrm{S})$, pero que también se aplica a otras relaciones categoriales, como $\mathrm{S}>\mathrm{S}$ «persona que ejerce habitualmente una actividad» (aduanero/a, bibliotecario/a pastor/a) y «persona estudiosa de una disciplina» (biólogo/bióloga, fisico/física, político/a). Con la intención de no complicar el diagrama arbóreo en los nombres de persona que desempeñan un oficio — que ya figuran desdoblados, cuando así lo requiere la categorización, en las clases adjetivo y sustantivo-, no desdoblaremos los nombres de oficio en cuanto a la moción genérica, salvo en aquellos casos donde la diferente datación de género supere los 50 años.

CONTAMinación. El proceso denominado contaminación (ingl. contamination, al. Kontamination, fr. contamination, it. contaminazione), que a primera vista parece similar al cruce léxico o blending, no responde a una creación intencionada o consciente de una nueva palabra, como ocurre en el blending, sino a un cruce fortuito e inconsciente por parte del hablante cuando dos palabras sinónimas, antónimas o parecidas formalmente «se activan simultáneamente sin querer y se realizan como una

11 Uno de los evaluadores me indica, a propósito de chico, $-a$, lo siguiente: «Queda claro que, desde esta perspectiva, en chica la - $a$ es morfema derivativo (y no flexivo), pero la -o de chico, ¿qué es: flexivo o derivativo?». Entiendo que también es morfema derivativo. Aquí sigo la propuesta de Booij (1996, 2012), que es la que sostienen también Haspelmath (2002) y Rainer (2004a), que consiste en distinguir entre la denominada flexión 'inherente' (plural y género de los sustantivos), y la flexión 'contextual' o sintácticamente inducida (plural y género de los adjetivos). Como señala Haspelmath (2002: 67), "gender in Italian and Spanish is an inflectional dimension of determiners, adjectives and verbs, but not of nouns. Nouns are all lexically associated with one of the two genders (masculine / feminine), but they are not morphologically marked for genders». 
única palabra» (Rainer 2007: 72) ${ }^{12}$. Con ejemplos tomados de Campos Souto (2008) ${ }^{13}$ : desparramar resulta de una contaminación de esparcir con derramar; apabullar resulta de una contaminación de apalear con magullar, etc.

CRUCE LÉXICO. (ingl. blending, al. Wortmischung, fr. mot-valise o amalgamation, it. parola macedonia) es un procedimiento de formación complejo que resulta de la combinación simultánea de dos procesos simples: dadas dos palabras independientes ${ }^{14}$, esto es, no relacionadas sintácticamente, una o ambas experimentan un proceso de reducción o truncamiento (ingl. truncation) para conformarse como coconstituyentes de la nueva palabra. La naturaleza compleja o combinada del procedimiento se percibe claramente si se contrastan dos formaciones como fotonovela y petroquímica. En la formación de fotonovela interviene un proceso simple de adición con dos bases de palabras preexistentes (composición), una de ellas, foto, resultante de otro proceso simple e independiente: el acortamiento de fotografía en foto. En la formación de petroquímica operan al tiempo la reducción o truncamiento de petróleo en petro- y la combinación de petro- con química: el constituyente petro- no existe como palabra autónoma, sino como constituyente para formar la palabra petroquímica. El procedimiento del cruce léxico es un proceso complejo que resulta de la combinación simultánea de dos procesos simples: la reducción de material fonológico de una de las palabras base o de ambas y la unión de los segmentos fonológicos resultantes.

Fradin (2015: 387) menciona cuatro razones que dificultan ofrecer una explicación adecuada del cruce léxico (blending) en la actualidad: a) como no se ajusta a pautas regulares de formación, su detección automática no es posible; los estudios rigurosos se basan en ejemplos atestiguados; b) el blending es muy dependiente de la estructura prosódica de la palabra en cada lengua, lo que obstaculiza las generalizaciones interlingüísticas; c) los blends que ofrece la bibliografía son muy heterogéneos, como también lo son las fuentes de donde provienen, y se mezclan con expresiones que no deberían catalogarse como tales; d) es dispar el grado de aceptabilidad que adjudican

$12 \quad$ La distinción entre estos procesos ya está presente en Pharies (1987: 275-279), bajo las denominaciones «Contamination» vs. «Blending» $\mathrm{y}$, como indica el propio Pharies, fue establecida con anterioridad por otros autores. En Pena (2018: 44) se nombra este proceso como cruce. Rainer lo denomina ‘contaminación’ y traduce el inglés blending como ‘cruce léxico'. En esta nueva versión se siguen las acuñaciones propuestas por Rainer.

13 La autora utiliza la etiqueta cruce léxico en clara sintonía con la tradición filológica hispánica y, más concretamente, con el $B D E L C$ y $D C E C H$ de Corominas donde se emplea la denominación cruce. Aquí, al igual que en la $B D M E$, optamos por denominaciones menos idiosincrásicas y, por tanto, más homologables en términos interlingüísticos.

14 Dada la escasez de blends resultantes de la reducción de más de dos palabras, me ceñiré a los blends formados sobre dos palabras base (source words). 
los hablantes a los ejemplares concretos. A estas cuatro razones se puede añadir una quinta: es práctica habitual en los estudios sobre el tema partir de unas premisas teóricas bastante estrictas que condicionan la búsqueda o confección del corpus, en lugar de trabajar con una selección preteórica de todas las palabras no analizables en morfos, tal como intenta hacer Thornton (1993:143) ${ }^{15}$.

El proceso del cruce léxico opera a la vez en el plano fonológico y semántico y no está determinado por patrones fijos ${ }^{16}$. Ello quiere decir que no hay una relación constante entre la fonología y la semántica del cruce léxico: el modo en que las subpartes o segmentos reducidos de las palabras base se combinan fonológicamente no repercute en su interpretación semántica (Fradin, Montermini \& Plénat 2009; Fradin $2015)^{17}$. Respecto a la parte formal, las pautas fonológicas no se corresponden con ningún esquema fónico recurrente: el punto donde acaba el primer constituyente y comienza el segundo está determinado por restricciones fonológicas (segmentales y prosódicas) al nivel de la sílaba o de sus constituyentes: ataque, núcleo, coda y rima (Plag 2003: 123-124; Fradin 2015: 394). En este sentido, la reducción que ocurre en las palabras base no tiene nada que ver con el acortamiento - como a veces se ha sostenido (Clas 1985, Lehrer 1996) - ya que las restricciones fonológicas que pesan sobre los acortamientos no son las que determinan el ajuste fonológico en los blends. La reducción operada en las palabras base es un mero truncamiento (Fradin 2015: 405-406). El acortamiento opera sobre una unidad léxica y denota la misma entidad $($ colegio $\rightarrow$ cole), mientras que el cruce léxico combina el significado de las palabras

15 Para la caracterización del cruce léxico, me basaré fundamentalmente en los siguientes estudios: Algeo (1977), Bauer (1983), Grésillon (1984), Clas (1985), Cannon (1986, 2000), Pharies (1987), Rainer (1993), Thornton (1993, 2004d), Casado Velarde (1999), Plag (2003), Gries (2004), Piñeros (2004, 2011), Renner (2006), Fradin, Montermini \& Plénat (2009), Léturgie (2011), Fradin (2015), Martín Camacho (2017).

16 Rainer (1993: 87-88) critica la definición de Pharies (1987: 227) para quien el blending en español se puede concebir como una subcategoría de la composición con un acortamiento simultáneo de una o ambas palabras. Son muchos los trabajos que se han inspirado en la definición de Pharies. Sin embargo, como afirma Rainer (1993), o más recientemente Fradin, Montermini \& Plénat (2009) y Fradin (2015), en el cruce léxico no se siguen los patrones formales y semánticos de la composición del español.

17 En Fradin, Montermini \& Plénat (2009: 39-41), y Fradin (2015: 402-404) se distinguen cinco patrones semánticos: 1) interpretación coordinada (fr. optimystique < optimiste + mystique, ingl. Oxbridge $<$ Oxford + Cambridge), 2) interpretación intersectiva (fr. autoimmobiliste $<$ automobiliste + immobile, ingl. fantabulous $<$ fantastic + fabulous, esp. brujeres $<$ bruja + mujeres), 3 ) interpretación argumental (fr. élevache $<$ élever + vache, ingl. sneakret $<$ to sneak + secret), 4) interpretación causal (fr. s'étrangueuler $<$ s'étrangler + s'engueuler), 5) interpretación ecuativa (fr. Chiruette $<$ Chirac + girouette). La mayor parte de los cruces léxicos o blends presentan una interpretación que encaja en uno de estos patrones semánticos. 
base de varias maneras y, por tanto, difícilmente puede ser deducible del significado de las palabras base.

Respecto al tamaño del cruce léxico (medido en número de sílabas) suele coincidir con el del constituyente más largo, que tiende a ser el segundo, aunque hay numerosos contraejemplos como los señalados por Rainer (1993) para el español: bocaperro $(<$ bocadillo + perro, o dictablanda (dictadura + blanda), etc. De todos modos, también es verdad, como veremos, que la palabra base más corta tiende a aparecer en primer lugar (perruquería < perro + peluquería). El acento siempre recae en el segundo miembro.

En la clasificación de los blends se suelen tener en cuenta tres factores formales: la reducción o truncamiento (ingl. truncation), la linealidad (ingl. linearization) y la superposición o solapamiento parcial de los constituyentes o palabras base (al. Überlappung, ingl. overlap, fr. superposition). La reducción puede afectar a los dos constituyentes o solo a uno. En inglés el blend prototípico está formado por el segmento inicial del primer constituyente y el segmento final del segundo (brunch $<$ br(eakfast $)+($ l)unch $)$, en español también siguen esta pauta bastantes formaciones (teleñecos $<$ tele(visión) + (mu)ñecos), pero es tanto o más rentable la formación de blends con reducción del primer segmento correspondiente a la primera palabra base, dejando intacta la segunda palabra base (cantautor $<$ cant(ante) + autor $)^{18}$. La linealidad ocurre cuando ninguna parte del blend correlacionado con la primera palabra base aparece intercalada a modo de infijo en las subpartes discontinuas pertenecientes a la segunda palabra base. La superposición se constata cuando se solapan segmentos fonológicamente similares, no necesariamente iguales (cf., por ejemplo, la proximidad entre /3/ y / $/$ en el fr. élévache (< élévage y vache) o entre $/ \mathrm{ro} / \mathrm{y} / \mathrm{lu} /$ en el esp. perruquería (< perro y peluquería). En el siguiente cuadro está sintetizada la clasificación tomando como referencia el de Fradin (2015: 394), quien a su vez lo adapta de Gries (2004: 646). La única novedad que ofrece el cuadro es que se incluye de manera sistemática el español, el francés y, ocasionalmente, el italiano ${ }^{19}$.

18 Lo mismo sucede en italiano, según Thornton (1993, 2004d) con los que ella denomina 'partial blends': «Questo tipo di formazioni [i.e. las formaciones tipo ing. smog, motel o brunch] in italiano è abbastanza raro [...]. In italiano è forte la tendenza a lasciare intatta la seconda parola che partecipa alla parola macedonia» (Thornton 2004d: 571). Parece, pues, que la construcción de blends a través de la reducción del primer integrante es, con diferencia, mucho más productiva en las lenguas románicas que en inglés.

19 Los segmentos de las palabras base que no figuran en el blend (aparecen entre paréntesis). Los segmentos superpuestos aparecen subrayados. Las abreviaturas deben leerse así: OV = overlap, LIN = linearization; trunc $=$ truncation . 


\begin{tabular}{|c|c|c|c|c|c|}
\hline & & A trunc $=$ ambos & $\mathrm{B}$ trunc $=1^{\circ}$ & $\mathrm{C}$ trunc $=\mathbf{2}^{\circ}$ & D trunc $=0$ \\
\hline \multirow{5}{*}{1} & \multicolumn{5}{|c|}{$+\mathrm{OV}+\mathrm{LIN}$} \\
\hline & ing. & & & & $\begin{array}{l}\text { sexploitation }<\text { sex }+ \\
\text { (ex)ploitation }{ }^{20}\end{array}$ \\
\hline & al. & & & wildschön $<$ wild + (bild)schön & $\begin{array}{l}\text { Paradiesel }<\text { Para }(\underline{\text { dies }}) \\
+ \text { Diesel }\end{array}$ \\
\hline & fr. & & & & $\begin{array}{l}\text { élevache }<\text { éle }(\underline{\text { vage })+} \\
\text { vache }^{21}\end{array}$ \\
\hline & esp. & & & & $\begin{array}{l}\text { perruquería (pelu)quería } \\
+ \text { perro }^{22}\end{array}$ \\
\hline \multirow{5}{*}{2} & \multicolumn{5}{|c|}{$+\mathrm{OV} \quad-\mathrm{LIN}$} \\
\hline & ing. & & $\begin{array}{l}\text { carnibbleous }<\text { car(niver) } \\
\text { ous }+ \text { nibble }\end{array}$ & & \\
\hline & al. & $\begin{array}{l}\text { dialügisch < día(lo)gisch } \\
+\underline{\underline{\text { ü}}(\mathrm{ge})}\end{array}$ & & & \\
\hline & fr. & & & & $\begin{array}{l}\text { autoimmobiliste }< \\
\text { auto(móvil)iste }+ \\
\text { immobile }^{23}\end{array}$ \\
\hline & esp. & & & & \\
\hline \multirow{5}{*}{3} & \multicolumn{5}{|c|}{$-\mathrm{OV}+\mathrm{LIN}$} \\
\hline & ing. & $\begin{array}{l}\text { brunch }<\text { br (eakfast })+ \\
\text { (l)unch }\end{array}$ & $\begin{array}{l}\text { Eurasia }<\text { Eur(ope })+ \\
\text { Asia }\end{array}$ & $\begin{array}{l}\text { smothercate }<\text { smother }+ \\
\text { (suffo)cate }\end{array}$ & \\
\hline & fr. & $\begin{array}{l}\text { plapier < pla(stique) }+ \\
(\text { pa)pier }\end{array}$ & $\begin{array}{l}\text { distribanque }< \\
\text { distributeur }+ \text { banque }\end{array}$ & $\begin{array}{l}\text { robotique }<\text { robot + (informat) } \\
\text { ique }^{24}\end{array}$ & \\
\hline & it. & tigone $<\operatorname{tig}(\mathrm{re})+($ le $)$ one & $\begin{array}{l}\text { polstrada }<\text { polizia }+ \\
\text { strada }\end{array}$ & & \\
\hline & esp. & $\begin{array}{l}\text { dictacracia }(<\text { dicta(dura }) \\
+(\text { demo }) \text { cracia })^{25}\end{array}$ & $\begin{array}{l}\text { bocaperro }<\text { boca }(\text { dillo }) \\
+ \text { perro }^{26}\end{array}$ & $\begin{array}{l}\text { robótica, aviónica, cablegrama } \\
<\text { cable }+ \text { (tele)grama }{ }^{27}\end{array}$ & \\
\hline
\end{tabular}

Tipología de blends con ejemplos en inglés, francés, español e italiano. Basado en Fradin (2015) y Gries (2004)

20 Otro ejemplo: slanguage $(<$ slang + (lang)uage $)$.

21 Otros ejemplos tomados también de Fradin, Montermini \& Plénat (2009): hippidémie (hippie + (épi) demie), métamourphose (< mét( $\underline{\text { amor }})$ phose + amour $)$, optimystique $(<$ opti( $\underline{\text { miste }})+\underline{\text { mystique }})$.

22 Otros ejemplos: $\underline{\text { brujeres }}(<\underline{\text { bruja }}+(\underline{\text { muje }})$ res $)$, cocacolonización $(<\operatorname{coca}(\underline{\text { cola }})+(\underline{\text { colo }})$ nización $)$,

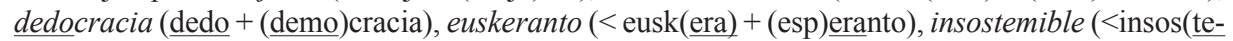

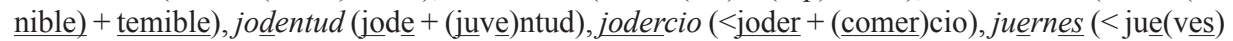
+ (vie)rnes), moncloaca $(<$ Moncloa $+(\underline{\text { cloa }}) \mathrm{ca})$, pechonalidad $(<$ pecho + (perso)nalidad), portuñol $<$ portu(gués) + (espa)ñol.

23 Otros ejemplos tomados de Fradin (2015:400): chérubambin (chéru(bin) + bambin), donde el segmento /chéru/ está separado del segmento /bin/ por una sílaba (bam/) que forma parte de la segunda palabra base.

24 Otros ejemplos: avionique (avion +(informat)ique), bureautique (bureau + (informa)tique).

25 Otros ejemplos: democradura ( demodura) (democra(cia) $\sim($ demo $)$ cracia + (dicta)dura), ofimática (< ofi(cina) + (infor)mática), teleñecos (tele(visión) + (mu)ñecos).

26 Otros ejemplos: cantautor $(<$ cant(ante) + autor $)$, crucidrama $(<$ cruci (grama) + drama), docudrama $(<$ docu(mento) + drama $)$.

27 Los cruces robótica y aviónica son préstamos del francés. Otros ejemplos: dedoñeco $(<$ dedo + (mu)ñeco), papamóvil (papa + (auto)móvil). 
Como se puede observar, la reducción de las palabras base (source words) puede ser consecuencia de suprimir algunos segmentos (fila 3) o el resultado de superponer ciertos segmentos o rasgos fonológicos (filas 1 y 2). No todas las casillas del cuadro corresponden a patrones generalizados ni intra ni interlingüísticamente (Fradin 2015: 395). Para muchos autores (Grésillon 1984, Cannon 1986, 2000, Plag 2003, Piñeros 2004, 2011), la superposición de los segmentos comunes en los márgenes de las palabras base es el fenómeno más extendido, el rasgo crucial y prototípico del cruce léxico o blend, especialmente los que combinan superposición (overlap) y linealidad $(+\mathrm{OV}+\mathrm{LIN})$.

En la superposición, además, los segmentos comunes no son el resultado del truncamiento de una de las secuencias idénticas o similares, sino precisamente el producto de dicha superposición (Fradin, Montermini \& Plénat 2009: 36, Piñeros 2011: 87). La superposición es la que promueve los blends intercalados (intercalative blends) (-LIN) pues no hay blends intercalados sin superposición.

Una idea certera que se cumple prácticamente en el cien por cien de los cruces del español con superposición y linealidad ${ }^{28}$ es la desarrollada en sus estudios por Eduardo Piñeros: el blend de este tipo (que él denomina associative blend) compacta o comprime las dos palabras base (source words) superponiendo la estructura de una de las palabras sobre la estructura de la otra y, como consecuencia, exhibe una fuerte tendencia a reproducir la estructura silábica y acentual de esta última (Piñeros 2004: 207-208), cf. loco + Colombia > Locombia 'Colombia como un país de locos', perro + peluquería $>$ perruquería 'peluquería para perros', pecho + personalidad $>$ pechonalidad $^{29}$.

Otra idea también muy acertada de este investigador, que por cuestiones de espacio no podemos desarrollar en esta nota, es el principio de «alineamiento de los márgenes» a la izquierda o a la derecha, basado en la similitud fonológica con una de las palabras base (Piñeros (2004: 215-219; 2011: 87-91). Utiliza, entre otros ejemplos, Modallín (< moda + Medellín) 'Medellín como un centro de moda', Naciomal $(<$ Nacional + mal) 'Nacional como un mal equipo de fútbol': «Modallín explota la afinidad fonológica entre moda y el inicio de Medellín, mientras que Naciomal aprovecha la afinidad fonológica entre mal y el fin de Nacional» (Piñeros 2011: 88).

$28 \quad$ Pueden consultarse, además del corpus de Piñeros (2011), los de Pharies (1987) y Rodríguez González (1989).

29 Y añade el autor otra idea muy pertinente y adecuada: «Another consequence of superimposing one source word upon the structure of the other one is that the segmental substring that the portmanteau seems to have lost from one of the source words corresponds exactly to the length of the other one, as if one word were covering/hiding part of the structure of the other word» (Piñeros 2004: 208). 
En varios estudios (Pharies 1987: 272; Thornton 1993: 146), Fradin, Montermini \& Plénat 2009: 34; Fradin 2015: 390) se destaca otra característica inherente de los cruces léxicos o blends: la de ser tipo hápax (ingl. hapax, nonce-word). Por referirnos al trabajo más reciente de los citados, Fradin (2015: 389-390) condensa tres propiedades destacadas de los blends, todas negativas: «a) no preservation of lexical integrity [...], b) no fixed pattern of compositionality [...], c) blends are type hapaxes. Unlike derived or compound units, they cannot form series». La naturaleza hapáctica del blend no debe entenderse literalmente en el sentido de formación registrada una sola vez, sino en el de formación que aparece muy ocasionalmente ${ }^{30}$, sobre todo si nos referimos a sectores específicos como el literario y el periodístico donde el acuñador del término desarrolla la función lúdica del lenguaje

La naturaleza hapáctica del blend viene determinada por la propia naturaleza del proceso: opera a la vez en el plano fonológico y semántico y no está determinado por pautas o patrones fijos, ya que intervienen bastantes variables en uno y otro plano sin correspondencias biunívocas, sino multívocas. Hay que tener en cuenta, además, que se trata de un proceso complejo que conlleva la realización simultánea de dos procesos simples: la reducción de material fonológico de una de las palabras base o de ambas y la unión de los segmentos fonológicos resultantes, frente a la composición que consiste en un proceso simple de adición de dos o más bases. Esta diferencia provoca que el tema o los temas reducidos o truncados del cruce léxico no estén incluidos en el léxico cuando se crean, sino que se originan en el momento de su acuñación, mientras que en la composición el tema acortado sí que está incluido en el diccionario (como tema de palabra inexistente) y disponible para ser reutilizado en la formación de nuevos compuestos.

Por consiguiente, si se parte de la premisa de que uno de los rasgos característicos del blend es que ninguno de los segmentos reducidos forma parte de series de palabras con el mismo segmento reducido como uno de sus constituyentes, habrá que admitir que ese segmento reducido se desgaje del blend en el que se acuñó por primera vez y comience a ser empleado de manera recurrente como tema acortado, sobre todo en posición inicial ${ }^{31}$, en la formación de nuevas palabras complejas, sobre todo compuestas:

30 A veces la distribución de las palabras base y del segmento reducido se repiten por analogía local con formaciones fruto de cruces léxicos preexistentes. Es lo que señala Rainer (1993: 89, 702) para la formación portuñol donde pudo influir la analogía con itañol o euskañol, o para la creación orteguiana venusmoto 'terremoto en Venus' bajo la influencia analógica de la pareja terreno y maremoto.

31 Raramente final, cf. -rragia ${ }^{2}(<$ hemorragia) en broncorragia, estomatorragia o -trón (< electrón) en ciclotrón, magnetrón, los dos galicismos (cyclotron, magnétron). 
cred(i)- < crédito (posiblemente del cruce léxico inicial credihogar, 1962): crediahorro, credicasa, credimoda, credimueble, credinegocio, credivuelo, etc.

exp(o)- < exposición (posiblemente del cruce léxico inicial expoferia, 1946): expociencia, expomueble, exponovia, expotaller, expotecnología, etc.

hidr(o)- < hidrógeno: (posiblemente del cruce léxico inicial hidrosulfuro, 1818): hidrocarburo, hidruro, hidracida, hidruro, etc. ${ }^{32}$

leuc(o)2- < leucocito (posiblemente del cruce léxico inicial leucemia, 1852): leucopenia, leucopoyesis, leucocidina, etc. ${ }^{33}$

narc(o)- $<$ narcotráfico (posiblemente de los cruces léxicos iniciales narcoterrorismo, narcoterrorista, 1982): narcoguerrilla, narcoavioneta, narcodinero, etc. petr(o)- < petróleo:) (posiblemente del cruce léxico inicial petroquímica, 1952): petrobolívar, petrodólar, petrocracia, petronegocio, petroprecio, petromonarquía, petromonarca, petrocasa, petrojuerga, etc.

La naturaleza de estas nuevas formaciones depende del estatus morfológico de elementos como los ejemplificados. Tanto Pharies (1987) como Rainer (1993) o Martín García (2017) hablan de afijos y, por tanto, de derivación. En la $B D M E$, siguiendo a Cottez (1980) los interpretamos en español como temas acortados, normalmente en primera posición, integrantes de compuestos especialmente (Pena 2008, 2018, Pena e Iglesias Cancela 2015, Iglesias Cancela 2018), al igual que Casado Velarde (1999) y Varela Ortega (2005). También los interpretan como integrantes de compuestos Fradin, Montermini \& Plénat (2009: 43) y Fradin (2015: 407), denominándolos respectivamente 'compositions cachées' y 'concealed compounding'.

El blending es un proceso que irrumpe con fuerza en la lengua inglesa durante el siglo XX y de ella irradia a las demás lenguas europeas, que importan tanto el proceso como muchas de las palabras resultantes de este. $Y$ no es un proceso utilizado en el léxico general, sino en sectores específicos: el lenguaje literario, el periodístico, especialmente en su vertiente política; desarrolla en ambos la función lúdica del lenguaje (playful language), ya que el acuñador del término crea, en connivencia con el interlocutor o lector, una aguda e ingeniosa asociación semántica entre los significados de las palabras base. Las creaciones dentro de estos dos ámbitos se caracterizan por su fugacidad: son formaciones que se registran ocasionalmente. Hay

\footnotetext{
32 Préstamos del fr. hydrosulfure, hydrocarbure, hydracide e hydrure (Cottez 1980, s.v. hydr(o)3-).

33 Los elementos acortados $h i d r(o) \_^{2}$ y leuc(o) $\_^{2}$ no existen como palabras. Este proceso, que cada vez es más productivo en el léxico de especialidad, se produce a costa de crear formantes homónimos de temas cultos, en este caso de origen griego: hidr(o)-1 «agua» (hidroterapia, hidrosoluble), leuc(o)- ${ }^{l}$ «blanco» (leucocito, leucorrea).
} 
otros dos sectores específicos donde tiene su origen el cruce léxico: el de la publicidad y el técnico científico, donde sí se mantienen de manera constante por su carácter interlingüístico, pero, como se acaba de indicar, pronto uno de los segmentos, el que se acuñó por primera vez — sobre todo el que figura en primera posición-, pasa a emplearse, de modo recurrente, en la formación de nuevas palabras complejas, sobre todo compuestas.

Por último, quisiera destacar aquellos casos que actualmente no se consideran cruces léxicos o blendings:

1) Los procedentes de sintagmas. En los primeros trabajos sobre el cruce léxico apenas se presta atención a si las bases del blending están relacionadas sintácticamente o no, esto es, si forman una sola unidad, un sintagma: cuernacionales $<$ cuernos nacionales, narraluces $<$ narradores andaluces, Servicaja $<$ Servicio de Caja. Hoy no se consideran cruces léxicos, sino sintagmas acortados, porque el significado del sintagma se mantiene (Rainer 1993: 90, Martín Camacho 2017: 119).

2) Tampoco son cruces léxicos los compuestos con palabras acortadas:fotonovela, bicicarril, pornoturismo, narcoterrorismo.

3) Los compuestos acortados, que son el resultado de acortamientos de un compuesto sintagmático preexistente en la lengua en los que uno o ambos constituyentes se han acortado: democristiano ( $<$ demócrata cristiano), baritenor ( $<$ barítono tenor); poli-mili ( $<$ político-militar), futsal $(<$ fútbol sala), mecatrónica $(<$ mecánica electrónica). Como muy bien indica Martín Camacho (2017: 115) son variantes diafásicas de los compuestos sintagmáticos con el mismo significado. En el mismo sentido se manifiesta Plag (2003: 122) a propósito de compuestos acortados como mocamp ( $<$ motor camp) motel $(<$ motor hotel) o sci-fi $(<$ science fiction).

También son compuestos los compuestos sintagmáticos acortados donde el proceso de acortamiento se ha combinado con la inversión de orden DoDe $>$ DeDo por influencia inglesa, por lo que el elemento determinado o nuclear está a la derecha (Casado Velarde 1999: 5085, Martín Camacho 2017: 115-116). Muchos de ellos son préstamos del inglés: ecodesarrollo (ingl. ecodevelopment $<$ ecological development), ecotasa ( $<$ ingl. ecotax $<$ ecological tax), ecoturismo $(<$ ingl. ecotourism $<$ ecological tourism), farmaindustria (< ingl. pharma industry < pharmaceutical industry); pero, una vez aceptado el patrón distribucional DeDo, se crean analógicamente en español nuevos compuestos de este tipo (Iglesias Cancela 2018): medicentro ( $<$ centro médico), electroauto (< auto eléctrico) $)^{34}$.

$34 \quad$ La estructura DeDo, con determinación a la izquierda, típica del inglés, se está extendiendo en las lenguas románicas. Vid. para el italiano Iacobini y Thornton (1992). 
4) Los acortamientos de compuestos preposicionales, también con la inversión indicada DeDo, en especial en variedades hispanoamericanas (Martín Camacho 2017: 116): infocentro $<$ centro de información, servicentro $<$ centro de servicios.

En las páginas precedentes he tratado de explicar cómo se han reinterpretado seis procesos morfológicos, con vistas a su aplicación en un base de datos morfoetimológica, la $B D M E$, accesible en https://bdme.iatext.es. Las modificaciones introducidas permiten precisar los tipos de procesos descritos en Pena (2018), trabajo íntegramente dedicado a describir la estructura y las propiedades de la $B D M E$.

\section{BIBLIOGRAFÍA}

Algeo, John (1977): «Blends. A structural and systemic view», American Speech 52, pp. 47-64. https://doi.org/10.2307/454719

ARONOFF, Mark (1994): Morphology by itself: stems and inflectional classes Cambridge Mass: MITT Press.

BAUER, Laurie (1983): English Word-formation. Cambridge: Cambridge University Press. https://doi.org/10.1017/CBO9781139165846

$B D E L C=$ Coromines, Joan (1973): Breve diccionario etimológico de la lengua castellana. Madrid: Gredos.

Bools, Geert (2012): The Grammar of Words: An Introduction to Linguistic Morphology. Oxford: Oxford University Press.

BooIJ, Geert (1996): «Inherent versus contextual inflection and the split morphology hypothesis», Yearbook of morphology, 1995, pp. 1-16.

CAmpos Souto, Mar (2008): «Morfología genética y etimología: los cruces léxicos», in Pilar Garcés (ed.), Diccionario histórico: nuevas perspectivas lingüisticas. Iberoamericana-Vervuert, pp. 41-63. https://doi.org/10.31819/9783865278678-004

CANNON, Garland (1986): «Blends in English word formation», Linguistics 24/4, pp. 725-753. https://doi.org/10.1515/ling.1986.24.4.725

Cannon, Garland (2000): «Blends», in Geert Booij, Christian Lehmann \& Joachim Mugdan (eds.) (2000-2002), vol. 1, pp. 952-956.

CASAdo Velarde, Manuel (1999): «Otros procedimientos morfológicos: acortamientos, formación de siglas y acrónimos», in Ignacio Bosque \& Violeta Demonte (dirs.), Gramática descriptiva de la lengua española. Madrid: Espasa, pp. 5075-5096.

CLAS, André (1985): «Composés lourds et créations brachygraphiques terminologiques», La Banque des mots 30, pp. 135-145.

CotTEZ, Henri (1980): Dictionnaire des structures du vocabulaire savant. Éléments et modèles de formation. Paris: Le Robert. 
DCECH = Corominas, Joan \& Pascual, José A. (1980-1991): Diccionario crítico etimológico castellano e hispánico. Madrid: Gredos. Edición electrónica en CD ROM.

$D E A=$ Seco, Manuel (dir.) (1999): Diccionario del español actual. Madrid: Aguilar. $D L E=$ Real Academia Española y Asociación de Academias de la Lengua Española: Diccionario de la lengua española. Edición del Tricentenario, 2014. http://dle.rae.es Don, Jan, Trommelen, Mieke \& Zonneveld, Wim (2000): «Conversión and category indeterminacy», in Booij et alii, Morphologie/Morphology: Ein internationales Handbuch zur Flexion und Wortbildung/An International Handbook of Inflection and Word Formation. Berlin/New York: Walter de Gruyter, vol. 1, pp. 943-952. DUE = Moliner, María (1998): Diccionario de uso del español actual. Madrid: Gredos. FrAdIN, Bernard (2015): «Blending», in Peter O. Müller et alii (eds.), Word Formation. An International Handbook of the Languages of Europe. Boston/Berlin: Mouton de Gruyter, vol. I, pp. 386-413.

Fradin, Bernard, Montermini, Fabio \& Plénat, Marc (2009): «Morphologie grammaticale et extragrammaticale», in Bernard Fradin, Françoise Kerleroux \& Marc Plénat, Aperçus de morphologie du français. Saint-Denis: Presses Universitaires de Vincennes, pp. 21-45.

GRÉSILlON, Almuth (1984): La Règle et le monstre: le mot-valise. Interrogations sur la langue, à partir d'un corpus d'Heinrich Heine. Tübingen: Niemeyer. https:// doi.org/10.1515/9783111352503

GRIES, Stefan Th. (2004): «Shouldn't it be breakfunch? A quantitative analysis of blend structure in English», Linguistics 42/3, pp. 639-667. https://doi.org/10.1515/ ling.2004.021

Haspelmath, Martin (2002): Understanding Morphology. London: Arnold.

IACoBInI, Claudio \& Thornton, Anna M. (1992): «Tendenze nella formazione delle parole nell'italiano del ventesimo secolo», in B. Moretti, D. Petrini \& S. Bianconi (eds.), Lineee di tendenza dell'italiano contemporaneo: atti del XXV congreso internazionale di studi della SLI. Roma: Bulzoni, pp. 25-55.

Iglesias Cancela, Yolanda (2018): Temas de palabras inexistentes. Diccionario de formantes y aplicación a la BDME. Tesis doctoral. USC: Escuela de doctorado Internacional.

LEHRER, Adrienne (1996): «Identifying and interpreting blends: an experimental approach». Cognitive Linguistics 7/4: pp. 359-390.

LÉTURGIE, Arnaud (2011): «Un cas d'extragrammaticalité particulier: Les amalgames lexicaux fantaisistes», Linguistica 51, pp. 87-104. https://doi.org/10.4312/ linguistica.51.1.87-104 
MARCHAND, Hans (1969²): The categories and types of present-day English wordformation. München: Beck.

MARCHAND, Hans, 1964: «A Set of Criteria for the establishing of derivational relationship between words unmarked by derivational morphemes», Indogermanische Forschungen, 69, pp. 10-19.

Martín CAmacho, José Carlos (2017): «La acronimia. Delimitación, rasgos generales y vitalidad de un procedimiento morfológico artificial», in Jesús Pena (ed.), Procesos morfológicos. Zonas de interferencia. Anejo 76 de Verba, Universidade de Santiago de Compostela, pp. 105-139.

Martín García, Josefa (2017): «Los límites de la prefijación», in Jesús Pena (ed.), Procesos morfológicos. Zonas de interferencia. Anejo 76 de Verba, Universidade de Santiago de Compostela, pp. 77-104.

$N G L E=$ RAE \& ASOCIACIÓN DE ACADEMIAS DE LA LENGUA ESPAÑOLA (2009): Nueva gramática de la lengua española. Madrid: Espasa.

Pena, Jesús \& Iglesias Cancela Yolanda (2016): «El tratamiento del léxico de especialidad en la BDME: problemas morfológicos», in Cecilio Garriga Escribano \& José Ignacio Pérez Pascual (eds.), Lengua de la ciencia e historiografia. Anejo 35 de la Revista de Lexicografía, pp. 231-248.

PenA, Jesús (1980): La derivación en español. Verbos derivados y sustantivos verbales. Universidade de Santiago de Compostela, Anejo 16 de Verba.

PenA, Jesús (1991): «La palabra: estructura y procesos morfológicos», Verba 18, pp. 69-128.

PENA, Jesús (1999): «Partes de la morfología. Las unidades del análisis morfológico», in Ignacio Bosque \& Violeta Demonte (dirs.), Gramática descriptiva de la lengua española. Madrid: Espasa, pp. 4306-4366.

PenA, Jesús (2008): «La creación del léxico científico y técnico», en Brea, Mercedes, Fco. Fdez. Rei e Xosé Luis Regueira (eds.): Cada palabra pesaba, cada palabra medía. Homenaxe ao profesor Antón Santamarina. Universidade de Santiago de Compostela: Servizo de Publicacións, pp. 163-170.

PENA, Jesús (2018): «La Base de datos Morfológica del español (BDME): Caracterización y estructura», in $\mathrm{M}^{\mathrm{a}}$ Pilar Garcés (ed.): Perspectivas teóricas y metodológicas en la elaboración de un diccionario histórico, Madrid/Frankfurt am Main: Iberoamericana/Vervuert, pp. 17-61. https://doi.org/10.31819/9783954879267-002

PenA, Jesús (2019): «La acumulación de significados morfológicos en la palabra derivada. A propósito de los nombres deverbales», en Ramón González Ruiz, 
Inés Olza y Óscar Loureda Lamas (eds.), Lengua, cultura, discurso. Estudios ofrecidos al profesor Manuel Casado Velarde, Ediciones Universidad de Navarra (EUNSA), pp. 685-701.

PhARIES, David (1987): «Blending in Spanish Word-Formation», Romanistisches Jahrbuch 38, pp. 271-289. https://doi.org/10.1515/9783110244946.271

PIÑEROS, Carlos-Eduardo (2004): «The creation of portmanteaus in the extragrammatical morphology of Spanish», Probus 16/2, pp. 203-240. https://doi.org/10.1515/ prbs.2004.16.2.203

PIÑERos, Carlos-Eduardo (2011): «¿Es el entrecruzamiento léxico realmente un proceso sustractivo?», Lingüistica Española Actual XXXIII/1, pp. 74-100.

PlaG, Ingo (2003): Word-Formation in English. Cambridge: Cambridge University Press. https://doi.org/10.1017/CBO9780511841323

Quirk, Randolph et al. (1980): A Grammar of Contemporary English. London: Longman.

RAINER, Franz \& WolBorSKa-LAURER, Joanna (2012): «El uso relacional del sufijo -dor/-dora en español y su relación con el francés», Romanische Forschungen 124, pp. 303-324. https://doi.org/10.3196/003581212802834832

RAINER, Franz (1993): Spanische Wortbildungslehre. Tübingen: Niemeyer. https:// doi.org/10.1515/9783110956054

RAINER, Franz (1999): «La derivación adjetival», in Ignacio Bosque \& Violeta Demonte (dirs.), Gramática descriptiva de la lengua española. Madrid: Espasa Calpe, pp. 4595-4643.

RAINER, Franz (2004a): «Premesse teoriche», in Maria Grossmann \& Franz Rainer (eds.): La formazione delle parole in italiano. Tübingen: Niemeyer, pp. 4-23.

RAINER, Franz (2004b): «Retroformazione», in Maria Grossmann \& Franz Rainer (eds.): La formazione delle parole in italiano. Tübingen: Niemeyer, pp. 493-497. https://doi.org/10.1515/9783110934410.493

RAINER, Franz (2007): «El papel de la analogía en la evolución de los patrones lexicogenésicos». Conferencia presentada en la Fundación Duques de Soria, Soria (julio de 2007).

RAINER, Franz (2008): «Inhibition of suffixation by suffix-like final strings in Spanish», in Bernard Fradin (ed.), La raison morphologique: hommage à la mémoire de Danielle Corbin. Amsterdam: John Benjamins, pp. 175-195. https:// doi.org/10.1075/lis.27.13rai 
RAINER, Franz (2012): «Escarceos sobre la conversión sustantivo $\rightarrow$ adjetivo en español», in Antonio Fábregas et al. (eds.), Los límites de la morfología. Estudios ofrecidos a Soledad Varela Ortega. Madrid: UAM Ediciones, pp. 369-382. https:// doi.org/10.15366/1.morfologia2012.022

RENNER, Vincent (2006): «Dépasser les déssacords: pour une approche prototypiste du concept d'amalgame lexical», in M. Pereiro \& H. Daniels (eds.), Le Désaccord. Nancy: Presses Universitaires de France, pp. 137-147.

Rodríguez GonzÁLez, Félix (1989): «Los cruces léxicos en el ámbito políticoperiodístico», Verba 16, pp. 357-395.

Sweet, Henry (1892): A new English grammar logical and historical, Part. I: «Introduction, phonology and accidence». Oxford: Clarendon Press.

Thornton, Anna M. (1993): «Italian blends», in Livia Tonelli \& Wolfgang U. Dressler (eds.), Natural Morphology: Perspectives for the Nineties. Padova: Unipresss, pp. 143-155.

THornton, Anna M. (1996): «On some phenomena of prosodic morphology in Italian: accorciamenti, hypocoristics and prosodic délimitation», Probus 8, pp. 81-112. https://doi.org/10.1515/prbs.1996.8.1.81

Thornton, Anna M. (2004a): «Mozione», in Maria Grossmann \& Franz Rainer (eds.): La formazione delle parole in italiano. Tübingen: Verlag, pp. 218-227.

Thornton, Anna M. (2004b): «Conversione», in Maria Grossmann \& Franz Rainer (eds.): La formazione delle parole in italiano. Tübingen: Verlag, pp. 499-553. https://doi.org/10.1515/9783110934410.499

Thornton, Anna M. (2004c): «Riduzione», in Maria Grossmann \& Franz Rainer (eds.): La formazione delle parole in italiano. Tübingen: Verlag, pp. 555-566. https://doi.org/10.1515/9783110934410.555

Thornton, Anna M. (2004d): «Parole macedonia», in Maria Grossmann \& Franz Rainer (eds.): La formazione delle parole in italiano. Tübingen: Verlag, pp. 567-571. https://doi.org/10.1515/9783110934410.567

VALERA, Salvador (2014): «Conversion», in Rochelle Lieber \& Pavol Štekauer (eds.) The Oxford Handbook of Derivational Morphology. Oxford: Oxford University Press, pp. 154-168.

Varela Ortega, Soledad (2005): Morfología léxica: la formación de palabras. Madrid: Gredos. 